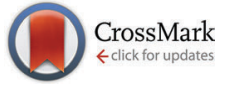

Cite this: J. Mater. Chem. B, 2015, 3, 5858

\title{
Fabrication of nanocellulose-hydroxyapatite composites and their application as water-resistant transparent coatings
}

\author{
Mai Ishikawa, ${ }^{a}$ Yuya Oaki, ${ }^{a}$ Yoshihisa Tanaka, ${ }^{b}$ Hideki Kakisawa, ${ }^{c}$ \\ German Salazar-Alvarez ${ }^{\text {de }}$ and Hiroaki Imai ${ }^{* a}$
}

\begin{abstract}
Nanosized composite rods $\sim 300 \mathrm{~nm}$ in length and $\sim 20 \mathrm{~nm}$ in width were produced by deposition of 22-77 wt\% of a c-axis-oriented hydroxyapatite (HA) on cellulose nanocrystals (CNCs). The CNCs functionalized with sulphonic groups were covered with the HA nanocrystals through controlled nucleation and growth under a moderately supersaturated condition in a solution system based on a simulated body fluid. Water-resistant transparent coatings 2-4 $\mu \mathrm{m}$ thick were obtained via evaporation-induced assembly of CNC-HA nanocomposites by casting their suspension on a glass substrate and the subsequent growth of HA nanocrystals by vapour hydrothermal treatment. The composite coatings exhibited improved mechanical strength compared to that of crustacean exoskeletons, and potential for bone regeneration.
\end{abstract}

Received 16th May 2015 Accepted 8th June 2015

DOI: $10.1039 / c 5 t b 00927 h$

www.rsc.org/MaterialsB adding inorganic compounds. In this manner, a biocomposite formed from CNCs and amorphous calcium carbonate nanoparticles was obtained at room temperature using the CNCs as nucleating substrates. ${ }^{8}$ However, the composite lacked the order and hierarchy found in natural biocomposites such as bones. ${ }^{9}$

Human bones are a nanoscopically ordered composite material consisting of $69 \mathrm{wt} \%$ of inorganic components and $22 \mathrm{wt} \%$ of organic components, such as collagen I. The dominant inorganic components are fibrous nanocrystals of hydroxyapatite (HA, $\left.\mathrm{Ca}_{10}\left(\mathrm{PO}_{4}\right)_{6}(\mathrm{OH})_{2}\right)$ that are precipitated between oriented collagen fibres with $c$-axis orientation. ${ }^{10}$ The hierarchical architecture of the nanocomposites provides great mechanical properties. Several composite materials based on HA nanocrystals have been reported to be possible bone substitutes. Titanium and stainless steel alloys are coated with HA to enhance osteoconductivity. ${ }^{11,12}$ Biodegradable polymers, such as polylactic acid and polycaprolactone, are employed as scaffolds for the composites. ${ }^{13-17}$ Simulated body fluid (SBF), which Kokubo invented in the $1980 \mathrm{~s}$, is a solution composed of the same inorganic ions as in human blood plasma and has been applied to the precipitation of HA on specific substrates and to evaluate the bioactivity of various biomaterials. ${ }^{18,19}$ Cellulose, especially bacterial cellulose, has received attention as a substitute for collagen in bone-repairing devices. However, the HA crystals in the composites were in the microscale and were not oriented like the biogenic architectures. ${ }^{20-22}$

The hammers of dactyl clubs called Odontodactylus scyllarus are composed of calcium phosphate and biopolymers. Crystalline and amorphous calcium phosphate particles are precipitated on helical chitin fibres of the exoskeletons, and the composite 
structure leads to extremely high strength. ${ }^{23,24}$ Thus, the composites of the crystalline calcium phosphate and polysaccharides, such as chitin and cellulose, are candidates for biodegradable, biocompatible, and high-strength materials. The main purpose of this work is to fabricate ordered nanocomposites with CNCs and HA. An SBF-based solution system was employed to precipitate nanosized HA on the surface of functionalised CNCs. The size and orientation of the HA crystals were controlled by varying the reaction conditions, such as the temperature, $\mathrm{pH}$, and molar ratio of calcium ions per functional groups on the CNC surface. Moreover, transparent water-resistant coatings were prepared on a substrate by casting the suspension of the nanocomposites and subsequent vapour-phase hydrothermal treatment. The novel nanocomposites could be the building units for functional materials in the field of biomedical applications and high mechanical strength films.

\section{Experimental}

\subsection{Synthesis of the nanocomposites}

In this study, to precipitate HA effectively, a simplified simulated body fluid (s-SBF), which removes unnecessary elements from the original elements, was employed. On the other hand, it is important when using SBF to maintain a suitable ionic strength, so that the concentrations of sodium ions and chloride ions are kept high. s-SBF was prepared in accordance with previous studies. ${ }^{25,26}$ Sodium chloride (Kanto Chemical), calcium chloride hexahydrate (Junsei Chemical), disodium hydrogen phosphate (Junsei Chemical), and hydrochloric acid (Junsei Chemical) were mixed to make the concentrations of sodium ions, calcium ions, chloride ions, and phosphate ions $141.0 \mathrm{mM}, 2.5 \mathrm{mM}, 165.0 \mathrm{mM}$, and $1.0 \mathrm{mM}$, respectively. The s-SBF solution was adjusted to a particular $\mathrm{pH}$ between 6.9 and 7.9 with the addition of tris(hydroxymethyl)aminomethane (Junsei Chemical). The preparation of a CNC dispersion and its surface modifications were also performed in accordance with the methods previously established. $^{8}$ The CNCs used for the study were derived from spruce and pine, and they were 5-10 nm wide and 100-300 nm long. Due to acid hydrolysis with sulphuric acid, the fibres had $\sim 0.34 \mathrm{mmol} \mathrm{g}^{-1}$ of surface sulphonic groups. We also used CNCs with carboxylate and hydroxy groups. The carboxylate groups were prepared via 2,2,6,6-tetramethylpiperidine 1-oxyl (TEMPO) oxidation. The hydroxy groups were prepared by heating the CNCs that had sulphonic groups. A certain amount of the CNC suspension was added to the precursor solution. The molar ratio of the calcium ions and sulphonic groups on the CNC surface was fixed between 30 and 230 with varied amounts of the CNCs in accordance with those in the previous study: the case of amorphous calcium carbonate. ${ }^{8}$ The s-SBF and CNC suspensions were put into an ultrasonic bath for $15 \mathrm{~min}$. After that, the mixtures were stirred in a thermostatic oven at $37{ }^{\circ} \mathrm{C}$ for $1 \mathrm{~h}$. The processes of ultrasound and stirring in the oven were repeated 1-3 times. The precipitated products were collected using centrifugation and washed several times with ethanol and pure water. The obtained samples were freeze-dried.

\subsection{Preparation of films of the nanocomposites}

The suspension synthesised at pH 7.9 was dropped onto superhydrophilic glass substrates that were treated with plasma etching in air for $10 \mathrm{~min}$. The substrates were set with leans of $20^{\circ}$. They were then dried at $60{ }^{\circ} \mathrm{C}$ for $3 \mathrm{~h}$, and transparent coatings were obtained. The solution deposition was repeated a certain number of times to increase the film's thickness. Vapour hydrothermal treatment was carried out to induce growth of the HA nanocrystals in the coatings. The hydrothermal treatment was performed by exposure of the samples to water vapour to prevent the degradation of the films by liquid water. The composite coating on the glass substrate was put into a small Teflon vessel $\left(20 \mathrm{~cm}^{3}\right)$. Then, the small vessel that contained the composite coating was put into a large Teflon vessel $\left(60 \mathrm{~cm}^{3}\right)$ with $10 \mathrm{~cm}^{3}$ of pure water. The Teflon vessels were placed in a stainless steel autoclave and then placed in the oven at $100-200{ }^{\circ} \mathrm{C}$ for $3-12 \mathrm{~h}$ for the vapour hydrothermal reaction. The coating was dried in a constant temperature oven at $60{ }^{\circ} \mathrm{C}$ in air.

\subsection{Characterisation of nanocomposites}

Fabricated coatings were observed using polarisation microscopy (Olympus BX51-FL) in a transmissive condition. The surface morphologies of the coatings were observed using scanning electron microscopy (SEM, JEOL JSM-7600F). The samples were coated with osmium $\sim 1 \mathrm{~nm}$ in thickness before observation. The composite nanorods and coatings were observed using transmission electron microscopy (TEM, FEI Tecnai $G^{2}$ F20). The composition of the samples was examined using TEM affiliated energy dispersive X-ray spectroscopy (EDX) and Fourier transform infrared spectroscopy (FTIR, JASCO FT/IR-4200). The crystallinity of the samples was analysed using X-ray diffraction (XRD, Rigaku MiniFlex II) using CuK $\alpha$ radiation. The content of the organic $\mathrm{CNC}$ and inorganic $\mathrm{HA}$ in the composites was determined using a thermogravimetric analysis (TGA) test with air using an SII TG/DTA 7200. The transmittance of the obtained coatings was investigated using spectrometry (JASCO V-670ST) in the visible light range. We observed the composite coatings under crossed nicols using polarized light microscopy to characterize the crystallinity of the films. The coating on the glass substrates was pinched with a wooden clip and half of the film was immersed in water for $4 \mathrm{~h}$ at $60{ }^{\circ} \mathrm{C}$ to test its water resistance. Nanoindentation tests were performed using a nanomechanical test instrument (Hysitron Ubi 1) furnished with a Berkovich diamond tip. Each sample was indented by $1000 \mu \mathrm{N}$. The hardness and Young's modulus were calculated from obtained load-displacement curves using the OliverPharr equation. ${ }^{27}$

\section{Results and discussion}

\subsection{Synthesis of the nanocomposites}

We used s-SBF for the preparation of the nanocomposites. In the absence of CNCs, no precipitates were collected in the aged solution at $37{ }^{\circ} \mathrm{C}$ for $3 \mathrm{~h}$ at $\mathrm{pH}$ 7.4. This means that the supersaturated solution is metastable to the deposition 

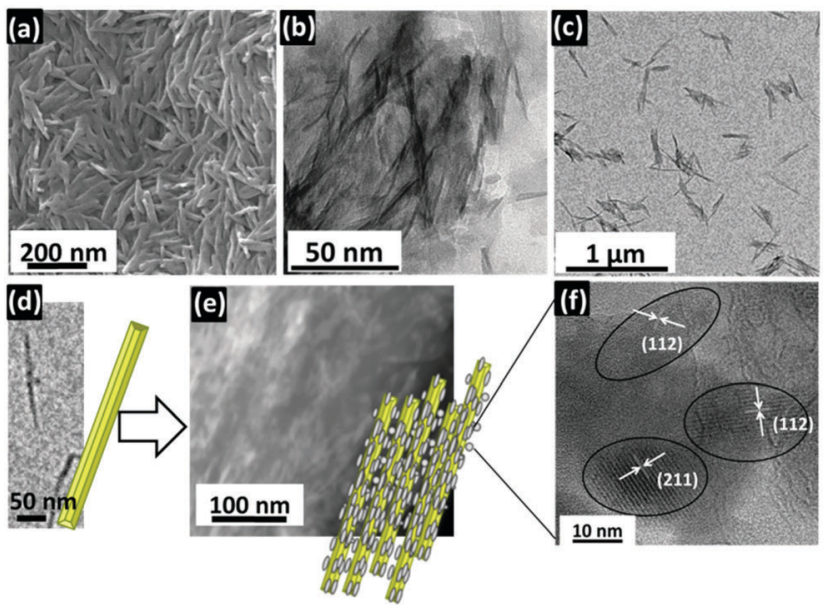

Fig. 1 A typical SEM image (a) and TEM micrographs (b, c) of the CNC-HA composites synthesised for $1 \mathrm{~h}$ in s-SBF at $\mathrm{pH} 7.9$ with a molar ratio of 40 of calcium ions and sulphonic groups. TEM images of the pristine CNCs (d), TEM image of the nanocomposites (e), and a high-resolution TEM image of the HA nanocrystals after the removal of CNCs with calcination at $400{ }^{\circ} \mathrm{C}$ in air (f).

of HA crystals under this condition. Fig. 1 shows the products collected in s-SBF with the CNCs that have sulphonic groups after being kept at $37{ }^{\circ} \mathrm{C}$ for $3 \mathrm{~h}$. The pristine CNCs were 5-10 nm wide and 100-300 nm long. ${ }^{8}$ After immersion for $3 \mathrm{~h}$, the width became approximately $20 \mathrm{~nm}$ by mostly covering the CNC fibril with fine particles of calcium phosphate of $\sim 5 \mathrm{~nm}$ in size. The inorganic part covering the CNCs was identified as HA according to the lattice image (Fig. 1f) and characteristic XRD signals due to the crystal lattice (Fig. 2a). As shown in Fig. 1e and f,
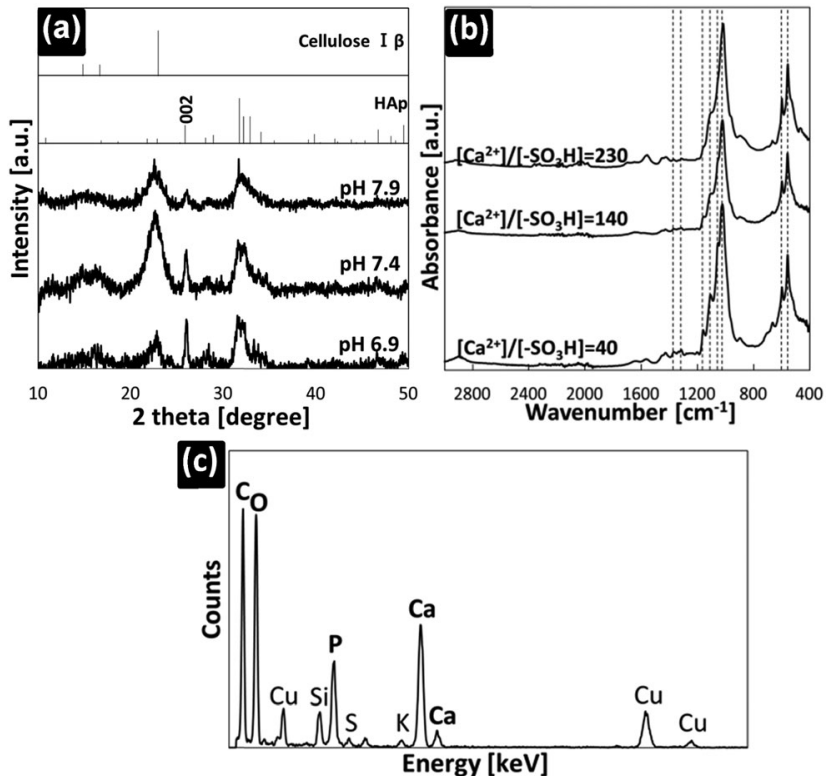

Fig. 2 XRD patterns with ICDD data of cellulose I $\beta$ (no. 00-056-1718) and HA (no. 00-055-0592) (a), FTIR spectra (b) and an EDX spectrum of the products collected in S-SBF with the CNCs having sulphonic groups. The molar ratio of the calcium ions and sulphonic groups was 40 in (a) and (c). The $\mathrm{pH}$ value was 7.9 in (b) and (c). the nanorods covered with the tiny HA nanocrystals were produced with a high degree of supersaturation at $\mathrm{pH}$ 7.9. The morphologies of the composites were varied by changing the $\mathrm{pH}$ of the s-SBF and the initial molar ratio of the calcium ions and the surface functional groups. The specific structure of the nanorods was formed with a high nucleation frequency due to the low solubility of calcium phosphate at pH 7.9. On the other hand, plate-like HA was gradually grown on the CNC fibrils at $\mathrm{pH} 6.9$.

As shown in the FTIR spectra (Fig. 2b), the absorption bands at 557 and 596, 1043, and $1087 \mathrm{~cm}^{-1}$ are assigned to the vibration, bending, and stretching modes of $\mathrm{PO}_{4}{ }^{3-}$ in $\mathrm{HA}$, respectively. ${ }^{28-30}$ They also suggest the presence of HA around the CNCs. The stretching vibration derived from $\mathrm{C}-\mathrm{O}\left(1016 \mathrm{~cm}^{-1}\right)$, the stretching vibration of $\mathrm{C}-\mathrm{O}-\mathrm{C}\left(1137 \mathrm{~cm}^{-1}\right)$, the wagging vibration of $\mathrm{CH}_{2}\left(1313 \mathrm{~cm}^{-1}\right)$, and the bending vibration of $\mathrm{C}-\mathrm{H}\left(1373 \mathrm{~cm}^{-1}\right)$ indicate that the composites contain HA and CNCs. ${ }^{31-33}$ The EDX spectrum also indicates all of the elements that were contained in the CNCs and HA. Thus, the nanocomposites were fabricated from the CNCs via coverage with the HA nanocrystals in the supersaturated solution. The $c$-axis of the HA was aligned along the long direction of the CNCs. Furthermore, the content of HA in the nanorods, which was determined using TGA, could be varied between 22 and $77 \mathrm{wt} \%$ by increasing the molar ratio of the calcium ions and the sulphonic groups.

Similar composites were obtained with the CNCs that had carboxylate and phosphate groups. On the other hand, deposition was not observed with CNC fibrils that had hydroxy groups in s-SBF. Thus, the deposition was induced in the metastable solution on the CNC surface with the sulphonic, carboxylate and phosphate groups. These facts suggest that anionic functional groups initiate the nucleation of HA and promote the formation of nanocomposites.

\subsection{Coatings of the CNC-HA nanocomposites}

Transparent coating of the nanocomposites was achieved by casting their suspension on a glass substrate. A superhydrophilic surface was needed to prepare the homogeneous, transparent films. As shown in Fig. 3, thin coatings with more than 90\% transmittance were successfully obtained using nanocomposites with 34 wt $\%$ of HA, which were highly dispersive in water. We obtained a single layer coating $\sim 2 \mu \mathrm{m}$ in thickness on the substrate. The film thickness increased to $\sim 10 \mu \mathrm{m}$ by 10 -layer coatings. The composite coatings were observed under crossed nicols using a polarized light microscope. Because extinction was observed on the composite coatings every $45^{\circ}$ during rotation, the coated nanocrystals were assembled in the same direction like a single crystal in the coatings. The $c$-axis of the fibrils was aligned parallel to the surface of the substrate and to the three-phase line consisting of air, the suspension, and the substrate. The (300) diffraction peak derived from HA was clearly observed on the coating of the CNC-HA composite rods. These results indicate that the $c$-axis of the HA that was made up of the nanocomposites was arranged parallel to the $c$-axis of the CNC fibrils.

The coating on the glass substrates was immersed in water for $4 \mathrm{~h}$ at $60{ }^{\circ} \mathrm{C}$ to test its water resistance. Unfortunately, the assembly of the nanocomposites turned to pieces in the water. 

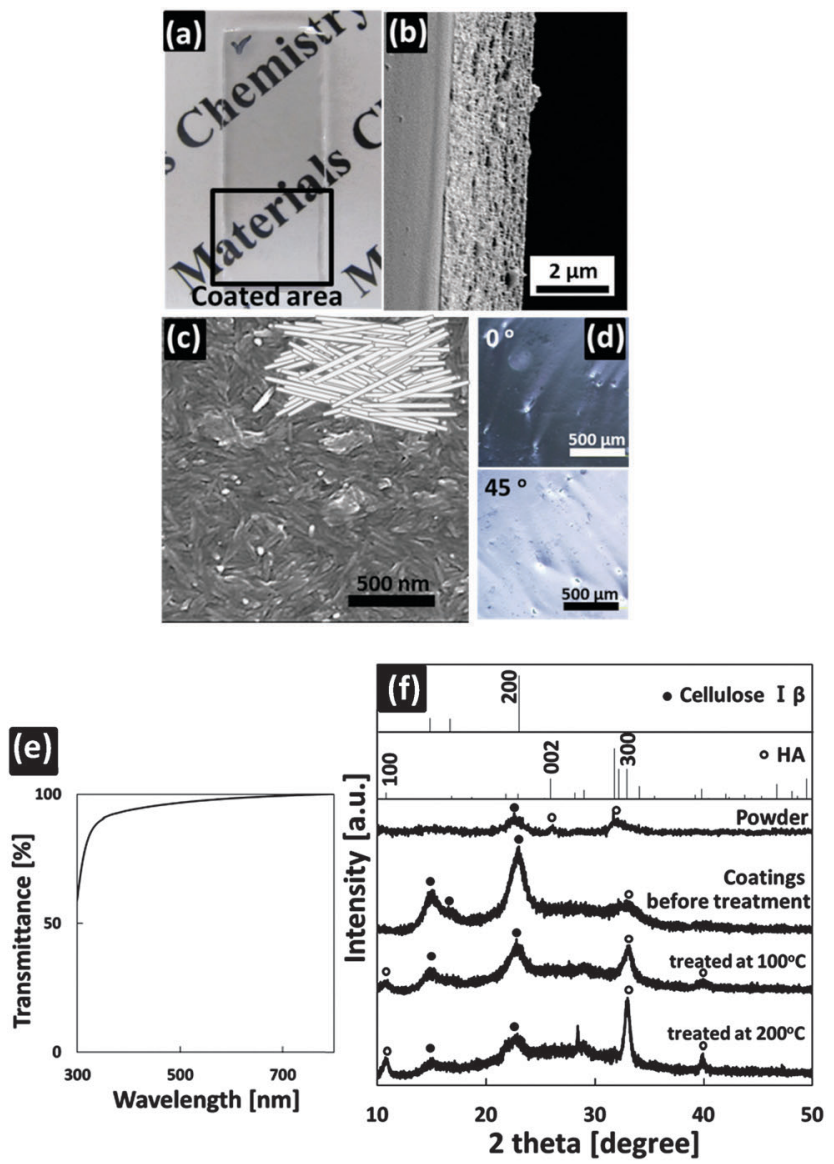

Fig. 3 Appearance (a), SEM images (b, c), polarised optical images (d), transmittance (e), and XRD patterns (f) of the coating of the nanocomposites.

Thus, we performed hydrothermal treatment in water vapour above $100{ }^{\circ} \mathrm{C}$ to improve the properties of the composite coatings.

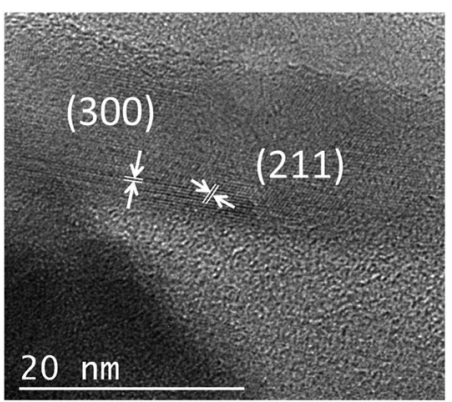

Fig. 4 A TEM image of the CNC-HA composite nanofibril in the coating after vapour hydrothermal treatment.
The hydrothermal treatment was performed by exposure of the samples to water vapour to prevent the degradation of the films by liquid water. The treated composite films showed tolerance to water at $60{ }^{\circ} \mathrm{C}$, although their transparent appearance did not change with the exposure to water vapour. As shown in Fig. 3, the (300) diffraction signal from HA increased after the treatment. The lattices of the (300) and (211) planes of the HA were clearly observed in the TEM image of the composite rod in the coating treated at $150{ }^{\circ} \mathrm{C}$ for $6 \mathrm{~h}$ (Fig. 4). Although the diffraction peaks due to the CNCs were relatively weakened with the increase in the signal intensity of the HA, the coating was not degraded with the treatment. These facts indicate that the HA nanocrystals covering the CNC core were grown in the presence of water vapour. The $c$-axis of the HA is deduced to elongate through the attachment of the nanocrystals. The durability of the coatings was enhanced by the interconnection of the HA crystals that covered the CNCs. Finally, we obtained water-resistant transparent coatings that consisted of the aligned CNC-HA nanocomposites.

\subsection{Properties of the composite coatings}

Crustacean exoskeletons are the nanocomposites of polysaccharides, such as chitin, and calcium carbonates or phosphates. The composite structures provide excellent mechanical properties. For instance, the hardness of the composites is commonly higher than that of conventional polymers. As shown in Table 1, the values of the hardness and the moduli of the

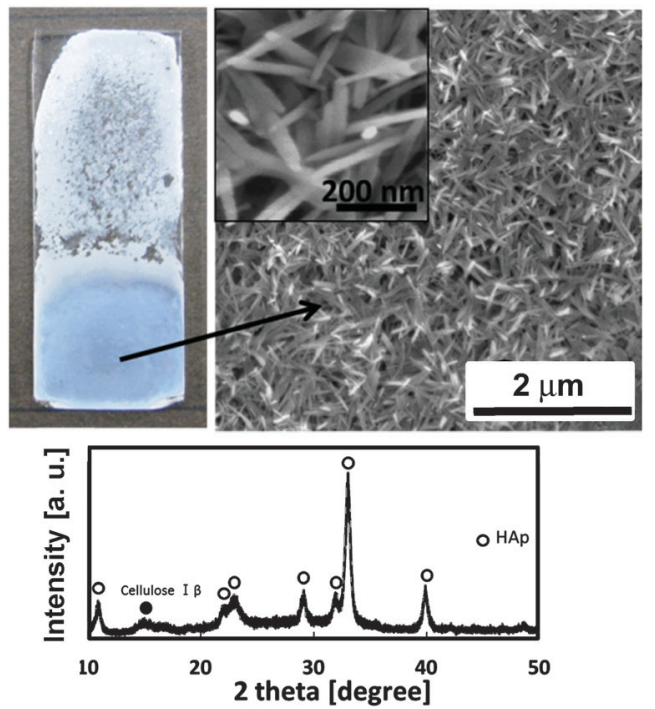

Fig. 5 Appearance, SEM images, and XRD profile of the nanocomposite coating after immersion in SBF for $24 \mathrm{~h}$.

Table 1 Mechanical properties of the coatings evaluated by nanoindentation ${ }^{a}$

\begin{tabular}{|c|c|c|c|}
\hline CNC-HA composite coating (before hydrothermal treatment) & $0.214 \pm 0.007$ & $21.9 \pm 0.7$ & $7.66 \pm 0.49$ \\
\hline
\end{tabular}

${ }^{a}$ Averages calculated by 5 -time measurements. ${ }^{b} 150{ }^{\circ} \mathrm{C}$ for $6 \mathrm{~h}$. 
composites are comparable to the organic-inorganic composites of lobsters and crabs. ${ }^{35-37}$ As compared with the Vickers hardness, the composite coatings are harder than general polymers.

The potential of mineralization on the materials' surface has been identified as an important phenomenon for bone-bonding capability implanted in bony defects. Kokubo proposed that a type of SBF may be useful for the estimation of the potential of mineralization that is the formation of a bone-like HA layer on the materials. ${ }^{18,19}$ In the present study, the water-resistant CNC-HA composite coatings were immersed in the s-SBF at pH 7.4 for $24 \mathrm{~h}$ to examine their bone conductivity. The composite coatings became opaque, and their thickness increased to $12 \mu \mathrm{m}$ with the deposition of the HA nanorods, 10-20 $\mathrm{nm}$ wide, on their surface as shown in Fig. 5. This indicates that the composite coatings have a higher potential for HA formation which relates the bone-bonding property of the designed materials for artificial bones. Thus, the transparent composite coatings could be applicable in the field of biomedical applications. Further biological evaluation is required to prove bone conductivity.

\section{Conclusions}

We produced nanometric composite rods that consisted of cellulose nanocrystals (CNCs) and hydroxyapatite (HA) by using a simplified simulated body fluid. Controlled nucleation with sulphonic and carboxylate groups as well as crystal growth under moderately supersaturated conditions are essential for fabricating the nanocomposites. Transparent thin coatings were successfully fabricated via evaporation-induced assembly of CNC-HA nanocomposites. The $c$-axes of the CNC and HA in the nanorods were aligned parallel to the surface. Water-resistant, transparent coatings of nanocomposites were achieved by elongating and interconnecting the HA nanocrystals with the vapour hydrothermal process. The composite coatings are expected to be a promising stage for fabricating novel organic-inorganic materials.

\section{Acknowledgements}

This work was partially supported by a Grant-in-Aid for Scientific Research (no. 22107010) on Innovative Areas of "Fusion Materials: Creative Development of Materials and Exploration of Their Function through Molecular Control" (area no. 2206) from the Ministry of Education, Culture, Sports, Science and Technology from Japan Society of the Promotion of Science and by a grant from the Wallenberg Wood Science Center (WWSC). The authors thank Christina Schütz for kindly providing the CNC materials and Prof. L. Berglund for fruitful discussions.

\section{Notes and references}

1 R. J. Moon, A. Martini, J. Nairn, J. Simonsen and J. Youngblood, Chem. Soc. Rev., 2011, 40, 3941.

2 A. Isogai, T. Saito and H. Fukuzumi, Nanoscale, 2011, 3, 71. 3 B. Wicklein and G. Salazar-Alvarez, J. Mater. Chem. A, 2013, 1, 5469.
4 Y. Habibi, L. A. Lucia and O. J. Rojas, Chem. Rev., 2010, 110, 3479.

5 N. Lin, J. Huang and A. Dufresne, Nanoscale, 2012, 4, 3274.

6 A. C. W. Leung, E. Lam, J. Chong, S. Hrapovic and J. H. T. Luong, J. Nanopart. Res., 2013, 15, 1636.

7 J. Yang, C. R. Han, X. M. Zhang and R. C. Sun, Macromolecules, 2014, 47, 4077.

8 D. Gebauer, V. Oliynyk, M. Salajkova, J. Sort, Q. Zhou, L. Bergström and G. Salazar-Alvarez, Nanoscale, 2011, 3, 3563 .

9 P. Fratzl and R. Weinkamer, Prog. Mater. Sci., 2007, 52, 1263.

10 M. Sadat-Shojai, M. Khorasani, E. Dinpanah-Khoshdargi and A. Jamshidi, Acta Biomater., 2013, 9, 7591.

11 S. Bauer, P. Schmuki, K. von der Mark and J. Park, Prog. Mater. Sci., 2013, 58, 261.

12 M. Vallet-Regi and J. M. Gonzalez-Calbet, Prog. Solid State Chem., 2004, 32, 1.

13 H. Kim, H. Lee and J. C. Knowles, J. Biomed. Mater. Res., Part A, 2006, 79, 643.

14 J. Chen, B. Chu and B. S. Hsiao, J. Biomed. Mater. Res., Part A, 2006, 79, 307.

15 X. Miao, D. M. Tan, J. Li, Y. Xiao and R. Crawford, Acta Biomater., 2008, 4, 638.

16 H. Kim, E. Lee, H. Kim, V. Salih and J. C. Knowlesb, Biomaterials, 2005, 26, 4395.

17 L. Shora, S. Güçeria, X. Wen, M. Gandhi and W. Sun, Biomaterials, 2007, 28, 5291.

18 T. Kokubo and H. Takadama, Biomaterials, 2006, 27, 2907.

19 T. Kokubo, S. Ito, Z. T. Huang, T. Hayashi, S. Sakka, T. Kitsugi and T. Yamamuro, J. Biomed. Mater. Res., 1990, 24, 331.

20 Y. Z. Wan, Y. Huang, C. D. Yuan, S. Raman, Y. Zhu, H. J. Jiang, F. He and C. Gao, Mater. Sci. Eng., C, 2007, 27, 855.

21 K. A. Zimmermann, J. M. LeBlanc, K. T. Sheets, R. W. Fox and P. Gatenholm, Mater. Sci. Eng., C, 2011, 31, 43.

22 K. Li, J. Wang, X. Liu, X. Xiong and H. Liu, Carbohydr. Polym., 2012, 90, 1573.

23 J. C. Weaver, G. W. Milliron, A. Miserez, K. Evans-Lutterodt, S. Herrera, I. Gallana, W. J. Mershon, B. Swanson, P. Zavattieri, E. DiMasi and D. Kisailus, Science, 2012, 336, 1275.

24 J. D. Currey, A. Nash and W. Bonfield, J. Mater. Sci., 1982, 17, 1939.

25 T. Kobayashi, S. Ono, S. Hirakura, Y. Oaki and H. Imai, CrystEngComm, 2012, 14, 1143.

26 W. Wang, Y. Oaki, C. Ohtsuki, T. Nakano and H. Imai, J. Asian Ceram. Soc., 2013, 1, 143.

27 W. C. Oliver and G. M. Pharr, J. Mater. Res., 1992, 7, 1564.

28 Y. Yusufoglu and M. Akinc, J. Am. Ceram. Soc., 2008, 91, 77.

29 G. Xu, I. A. Aksay and J. T. Groves, J. Am. Chem. Soc., 2001, 123, 2196.

30 Y. Lee, E. Yeong, M. Hahm, E. S. Matsuya, M. Nakagawa and K. Ishikawa, J. Mater. Sci., 2007, 42, 7843.

31 S. Koutsopoulos, J. Biomed. Mater. Res., 2002, 62, 600. 
32 M. Fan, D. Dai and B. Huang, Fourier Transform - Materials Analysis, 2012, ISBN 978-953-51-0594-7.

33 D. Ciolacu, F. Ciolacu and V. I. Popa, Cellul. Chem. Technol., 2011, 45, 13.

34 M. Chanda and S. K. Roy, Plastics Technology Handbook, CRC Press, 4th edn, 2006.
35 T. Saito, Y. Oaki, T. Nishimura, A. Isogai and T. Kato, Mater. Horiz., 2014, 1, 321.

36 C. Sachs, H. Fabritius and D. Raabe, J. Mater. Res., 2006, 21, 1987.

37 B. W. Cribb, A. Rathmell, R. Charters, R. Rasch, H. Huang and I. R. Tibbetts, Arthropod Struct. Dev., 2009, 38, 173. 